\title{
Establishment of a New Equation for Ultrasonographic Estimated Foetal Weight in Chongqing: a Prospective Study
}

Chenhuizi Wu ( $\sim$ hz18883936094@sina.com )

The Second Affiliated Hospital of Chongqing Medical University https://orcid.org/0000-0002-9016$488 \mathrm{X}$

\section{Jianfeng Sun}

The Second Affiliated Hospital of Chongqing Medical University

\section{Xiaojing Dong}

The Second Affiliated Hospital of Chongqing Medical University

\section{Liuyun Cai}

The Second Affiliated Hospital of Chongqing Medical University

\section{Xinru Deng}

The Second Affiliated Hospital of Chongqing Medical University

\section{Fenglan Zhang}

The Second Affiliated Hospital of Chongqing Medical University

\section{Yang Shu}

The Second Affiliated Hospital of Chongqing Medical University

Maochao Zhang

The Second Affiliated Hospital of Chongqing Medical University

\section{Xiaodong Luo}

The Second Affiliated Hospital of Chongqing Medical University

\section{Research Article}

Keywords: Estimated foetal weight, Prenatal ultrasound, Chongqing foetuses, Regression equation, Birth weight

Posted Date: January 11th, 2022

DOI: https://doi.org/10.21203/rs.3.rs-973686/v1

License: (c) (i) This work is licensed under a Creative Commons Attribution 4.0 International License. Read Full License 


\section{Establishment of a new equation for ultrasonographic estimated foetal} weight in Chongqing: a prospective study

Chenhuizi $\mathrm{Wu}^{1}$, Jianfeng Sun ${ }^{2}$, XiaoJing Dong ${ }^{1}$, Liuyun $\mathrm{Cai}^{1}$, Xinru Deng ${ }^{1}$, Fenglan Zhang $^{1}$, Maochao Zhang ${ }^{1}$, Xiaodong Luo ${ }^{1^{*}}$

Corresponding Author: Xiaodong Luo

Email: Luoxiaodong@hospital.cqmu.edu.cn

Department of Obstetrics and Gynaecology, the Second Affiliated Hospital of Chongqing Medical University, Chongqing, Chongqing 400010, People's Republic of China.

A full list of author information is available at the end of the article.

Abstract

Background: Variations in foetal growth between populations should not be ignored, and a single universal standard is not appropriate for everyone. Therefore, it is necessary to develop a new ultrasound estimation equation that adapts better to regional population characteristics. The purpose of this study was to create a new equation for ultrasound estimation of foetal weight according to the local population in Chongqing and compare it with representative equations.

Methods: This prospective study included data on pregnant women who gave birth to a child at full term in our hospital from December 2016 to November 2019. Foetal ultrasound parameters included biparietal diameter (BPD), head circumference (HC), abdominal circumference $(A C)$, and femur diaphysis length (FDL). The foetal weight compensation model was established by using the second-order linear regression 
model, and then, the foetal weight equation was established by utilizing the multiple reverse elimination regression technique. Last, the absolute error and relative error were used to compare the accuracy of the equations established in this study with representative equations.

Results: Through the foetal weight compensation equation, the new equation suitable for Chongqing foetuses was successfully established with the variables of BPD, HC, AC, and FDL. The following foetal weight prediction equation was $\begin{array}{lll}\text { established } & \text { in this study: }\end{array}$ $\log _{10}(E F W)=3.002741+0.00005944 *\left(B^{\prime} D^{\wedge} 2\right)+0.00000222^{*}\left(H C^{\wedge} 2\right)-0.000002078^{*}\left(A^{\wedge}\right.$ $2)+0.00004262 *\left(F D{ }^{\wedge} 2\right)-0.008753 * B P D-0.000884 * H C+0.003206 * A C-0.002894 * F D L$ (BPD: mm; HC: mm; AC: mm; FDL: mm). In the sets established by the 1925 data, the mean absolute error and standard deviation of the estimation error of the new equation were $178.9 \mathrm{~g}$ and $140.3 \mathrm{~g}$ respectively. In the validation sets established with 300 data points, the mean absolute error and standard deviation of the new equation were $173.08 \mathrm{~g}$ and $128.59 \mathrm{~g}$ respectively. Compared with representative equations, the mean absolute error and the standard deviation of the new equation were the lowest. The equation established in this study better predicted foetal weight $(\mathrm{P}<.001)$.

Conclusions: According to the local population characteristics of Chongqing, this study created a foetal weight estimation equation that is more accurate and suitable. This equation is clinically valuable for the monitoring and management of foetal weight. 
Key words: Estimated foetal weight, Prenatal ultrasound, Chongqing foetuses, Regression equation, Birth weight

\section{Background}

Estimated foetal weight (EFW) is an important part of antenatal care, and foetal weight is a key factor influencing the timing, manner and perinatal outcome of foetal delivery[1-2]. With the development of ultrasound technology, ultrasound estimation of foetal weight before birth has become one of the principal means for modern obstetrics to assess foetal weight, and identify and address high-risk pregnancies[3-5]. To obtain a good perinatal outcome, foetal weight can be dynamically monitored by ultrasound. Based on foetal biological parameters measured by ultrasound, scholars at home and abroad have derived several regression equations for EFW by multiple linear regression methods[6-10].

Most hospitals in China still use the Hadlock equation established in 1985 based on the US foetal database[7], probably because ultrasonic diagnostic instruments mostly default to this equation. Due to regional environments, racial differences[11], genetics, dietary factors[12] and other factors[13], the trunk ratio and fat content of foetuses in different regions may vary. Variations in foetal growth between populations should not be ignored. There may be some error in directly using the Hadlock equation to estimate foetal weight in Chinese foetuses. In recent years, the ultrasonographic equation suitable for Chinese Han population was also being explored in China to predict foetal weight[14-15]. Chongqing is the largest industrial and commercial city in southwest China. Chongqing has many ethnic groups and 30 million permanent residents[16]. Chinese residents have quite different physical conditions, due to 
differences in geography, ethnicity, socioeconomic status, and the living environment[17]. For example, the average height of Beijing residents is higher than that of Chongqing residents[18]. Therefore, to improve the accuracy of estimating foetal weight in the local population, it is necessary to identify a new and more accurate regression equation to estimate foetal weight according to the population characteristics of each different regions.

The aim of this study was first to establish the weight compensation equation, to make the ultrasonic measurement correspond to the foetal weight at the time of ultrasonic measurement and reduce the estimation error of the model. Then, we established the ultrasonographic estimation equation of foetal weight based on foetal growth parameters of pregnant women in Chongqing and compared this equation to the Hadlock equation[7], Combs equation[8] and Stirnemann equation[9] to ensure the accuracy of the new equation.

\section{Methods}

This prospective study included the data of pregnant women who gave birth to a single child at full term in our hospital from December 2016 to November 2019. This study recruited women whose gestational weeks ranged from 37 weeks to $41+6$ weeks. All of them had a single gestation and the neonates were born with normal outcomes. All ultrasound examinations were performed using GE Voluson E8 and GE Voluson E10 machines (General Electric Healthcare, U.S.A). The birth weights of all foetuses were determined with the electronic baby scale DY-1 (Shanghai Guangzheng Medical Instrument Co., LTD). This study involved 5 sonographers. All the sonographers received specific training, and their measurement techniques were subjected to rigorous quality assurance[19-20]. 
The inclusion criteria were as follows: pregnant women in various districts and countries in

Chongqing; women who had a definitive knowledge of the gestational week (the definite last menstrual period was consistent with the gestational week corresponding to the crown-rump length measured by ultrasound); and women whose ultrasound examination was performed within 7 days before delivery. There was no limit to the number of pregnancies.

The exclusion criteria were as follows: those with a structural foetal anomaly detected on ultrasound and patients who had complications during pregnancy (including gestational diabetes, gestational hypertension, and foetal growth restriction).

General information included maternal demographic data (ethnicity and age), data of the last menstrual period, pregnancy complications, gestational week at birth, birth data, ultrasonic examination dates, and new-born birth weight (each new-born was placed on an electronic scale after delivery by a senior nurse working in the delivery room) .

Foetal ultrasound parameters included the biparietal diameter (BPD), head circumference (HC), abdominal circumference (AC), and femur diaphysis length (FDL), all of which were measured in the last prenatal ultrasound.

The measurement methods were as follows[21-22]: foetal growth parameters were measured three times, and the mean values were taken. Foetal BPD and HC measurements were taken in a cross-sectional view of the foetal head at the level of the thalami, with an ideal angle of insonation of 90 degrees to the midline echoes. The appearance of both hemispheres was symmetrical. The continuous midline echo (falx cerebri) was broken in the middle by the cavum septi pellucidi and thalamus. The cerebellum was not visualized in the section. Linear distance was measured from the outer edge of the proximal to the outer edge of the distal skull. Foetal HC 
containing no extracranial soft tissue along the outer edge of the foetal skull was directly measured using the elliptical function key.

Foetal AC measurements were taken in a transverse-section view of the foetal abdomen as circular as possible, showing the stomach bubble, and umbilical vein at the level of the portal sinus. The kidneys and bladder did not have to be visible. The AC was measured at the outer surface of the skin line, directly with ellipse callipers.

Foetal FDL was imaged optimally, with both ends of the ossified metaphysis clearly visible. The longest axis of the ossified diaphysis was measured. An angle between the femur and insonating ultrasound beams between 45 degrees and 90 degrees was acceptable. Each calliper was placed at the ends of the ossified diaphysis without including the distal femoral epiphysis if it was visible. This measurement excluded triangular spur artefacts.

\section{Statistical analysis}

To make the ultrasonic measurement correspond to the foetal weight at the time of ultrasonic measurement, a weight compensation equation was established. Taking gestational age at ultrasonography as the independent variable and birth weight as the dependent variable, a second-order linear regression model was used to establish the weight compensation equation for the changes with time to compensate for the difference between the weight and the birth weight during ultrasonic measurement at the time of ultrasonic measurement. The foetal weight prediction model was established by reverse elimination regression after compensating for the weight difference[23].

All measured values (BPD/HC/AC/FDL) were included, including first-order, second-order, and 
interactive terms (e.g., $A C, A C \times A C, A C \times H C$ ), and the nonsignificant parameters were then deleted until the significance of all parameters reached .01 . This equation was then compared with the Hadlock equation, Combs equation and Stirnemann equation by estimation error.

Data not involved in model establishment were collected as a validation set to verify the accuracy of different equations. The statistical software package IBM SPSS 24.0 (IBM Inc., New York) was used for the data analyses.

\section{Results}

A total of 2000 clinical data points were collected, of which 1925 foetal data points were eligible for screening after post hoc exclusion. These women were 19 to 35 years old, with an average age of 29 years. The mean time of ultrasound examination was 2.44 days before delivery. To reduce the estimation error of the model, the following foetal weight compensation equation was established in this study: $\nabla$ Weight $=-35534.421687+1909.952928^{*} \nabla$ GA-23.412541*( $\nabla$ GA^2) (Weight unit: g; gestational age unit: weeks). Compensation weight was the difference between the time of birth and the time of ultrasound examination in the weight compensation formula.

Through the foetal weight compensation equation, the weights data of 1925 foetuses were modified to obtain the weight measured by ultrasound. Table 1 shows the fitting equation of the foetal weight estimate and their correlation coefficients ( $R 2=0.974)$ as well as the standard error. Then, reverse knockout regression was used to establish the Chongqing foetal weight prediction equation: 
$\mathrm{mm}$; FDL: $\mathrm{mm}$ ).

The newly established equation was compared with three representative weight prediction equations, which were proposed by Hadlock, Combs and Stirnemann et al. Table 2 displays the comparison of the establishment methods for the four equations. The four equations were all multiple parameter evaluation equations, except the foetal $\mathrm{HC}$ measurement method, which was slightly different, and the measurement method of other indicators was the same. The results might be somewhat affected but comparable.

First, this study compared the estimated errors of the four equations for each gestational week (Figure 1). This estimation error was cited as the difference between the estimated weight and the compensated weight. Only when the pregnancy was 37 weeks was the mean absolute error and standard deviation of the Chongqing equation not the least among the four equations. It was found that the mean absolute error values of the Chongqing equation established in this study were respectively $179.06 \mathrm{~g}, 171,63 \mathrm{~g}, 175.88 \mathrm{~g}, 192.20 \mathrm{~g}$ and $174.54 \mathrm{~g}$ for gestational weeks 38 to 42 and were the smallest error values among the four equations in each gestational period. The standard deviations of the Chongqing equation were respectively $139.14 \mathrm{~g}, 136.76 \mathrm{~g}, 146 \mathrm{~g}$, $136.57 \mathrm{~g}$ and $126.64 \mathrm{~g}$ for gestational weeks 38 to 42 and were the smallest standard deviations among the four equations in each gestational period.

Next, we compared the estimated errors of the four equations again (Figure 2). This estimation error was cited as the difference between the estimated weight and the birth weight. The estimated error $\pm 1.96 \mathrm{x}$ standard deviation was interpreted as $95 \%$ error within this range. The estimated weight per gestational week estimated by the Chongqing equation at 37 to 42 weeks of gestation was the closest of the four equations to the actual average weight, while the weight 
estimation equation established by Hadlock and Combs had the largest error.

To further verify the accuracy of the Chongqing equation, gestational age was not considered, and the mean absolute error and standard deviation of the estimation error of the equation established in this study were $178.9 \mathrm{~g}$ and $140.3 \mathrm{~g}$ respectively (Table 3 ). The new equation had the smallest estimation error and the smallest standard deviation, and the new equation was more accurate for the prediction of foetal weight in Chongqing $(\mathrm{P}<.001)$.

Another 300 foetal data points were collected to verify the accuracy of the new equation, and they constituted the validation sets. These pregnant women were aged from 18 to 40 years, with an average age of 28 years. The mean time of ultrasound examination was 0.84 days before delivery. Table 4 shows that the mean absolute error and standard deviation of the estimation error of the Chongqing equation were $173.08 \mathrm{~g}$ and $128.59 \mathrm{~g}$ respectively, both of which were the smallest among the four equations in the validation sets. Therefore, the application of this equation to the clinical EFW would obtain a better result $(\mathrm{P}<.001)$.

\section{Discussion}

Prenatal ultrasound can accurately predict foetal weight to detect giant infants in a timely manner, reduce unnecessary trial delivery, and avoid maternal and infant injuries such as cervical laceration, shoulder dystocia, and bone and brachial plexus injury[24]. It can also reduce the rate of selective caesarean caused by incorrect EFW and insufficient confidence of pregnant women in a trial of delivery[25]. In addition, prenatal ultrasound can identify very-low-birth-weight infants and fully estimate the foetus's tolerance to hypoxia during contractions so that the doctor can choose timely caesarean section to deliver the pregnancy and avoid adverse perinatal 
outcomes[26-27].

Both Kiserud et al.[5] and Gardosi et al.[28] showed that there are normal physiological changes between different countries and nations and that a single universal standard is not appropriate for everyone. Therefore, the purpose of this study was to establish the weight compensation equation, which is used to make the ultrasonic measurement correspond to the foetal weight at the time of ultrasonic measurement and reduce the estimation error of the model. Then, we created and verified the optimal regression equation for predicting foetal weight, according to the population characteristics of Chongqing.

Because the timing of delivery was uncontrollable, prenatal ultrasound was performed every 7 days after 37 weeks of gestation. The ultrasound examination time was extended to 7 days. Previous studies[5-7] ignored the difference between ultrasonic measurement time and birth time and directly used the data measured during the last ultrasonic examination to correspond to birth weight to calculate estimated foetal weight, which would result in a large estimate. The relationship between gestational age and birth weight was established by a nonlinear regression equation. For example, we can use the weight compensation equation to calculate the foetal weight at 37 weeks of gestation, when the ultrasound examination is performed at 37 weeks of gestation and the foetus is born at 38 weeks of gestation. A weight compensation mechanism was established to compensate for the difference between the weight measured by ultrasound and the birth weight.

We found that the mean absolute error values of the Chongqing equation established in this study were respectively $179.06 \mathrm{~g}, 171,63 \mathrm{~g}, 175.88 \mathrm{~g}, 192.20 \mathrm{~g}$ and $174.54 \mathrm{~g}$ from 38 to 42 weeks of gestation and that these error values were the smallest of the four equations in each 

standard deviations among the four equations in each gestational period. Figure 1 shows that except for 37 weeks, the estimation error of the Chongqing equation was the smallest all other gestational weeks. This might be because there were data from only 63 pregnant women were collected at $\mathbf{3 7}$ weeks, making this a small sample size. Further discussion is needed. compensation mechanism. The accuracy of the new equation and other representative equations was prospectively verified by comparing the estimation error. We analysed not only the estimated error of each gestational week but also the overall estimated error. The estimation error was the error of the estimation weight and compensation weight and the error of the estimation weight and birth weight. The estimated weight per gestational week estimated by the Chongqing equation at 37 to 42 weeks of gestation was the closest of the four equations to the actual average birth weight. In the sets established by the 1925 data, the mean absolute error and standard deviation of the estimation error of the equation established in this study were mean absolute error and standard deviation of the Chongqing equation were $173.08 \mathrm{~g}$ and $128.59 \mathrm{~g}$, respectively. The equation established in this study was the most accurate in predicting weight estimation equation established in this study was more applicable to Chongqing foetuses, and it could be used to obtain a more accurate foetal weight estimation of Chongqing foetuses. 
geographic factors and used the multiple reverse elimination regression technique and the ten-fold cross verification method to establish the foetal weight prediction model. The method for deriving the equation can be extended to China and other parts of the world to establish a suitable reference equation for local foetal weight estimation. Second, we collected a large amount of data, specifically data from 1925 cases. They were carefully screened to ensure good health, excluding ethnic, geographic and other factors. Foetal structural abnormalities that may lead to adverse pregnancy outcomes were excluded, and patients with pregnancy complications were also excluded, as all of these factors may affect the growth rate and development of the foetus, and affect the derivation and establishment of this formula. For example, the inclusion of gestational diabetes may increase the incidence of premature foetuses and macrosomia[29], leading to a decrease in the uptake of the equation. Finally, from the analysis of the comparative results, the equation established in this study had the least error and was most suitable for the prediction of Chongqing foetal weight. Compared with the representative equation, it was proven that the application of this equation had high clinical guidance and reference value for the monitoring and management of foetal weight.

The limitation of this study is that the sonographers could see the data automatically display on the screen after performing the measurements, which may lead to the deviations from the expected value. Although we recruited well-trained ultrasound operators specifically instructed by the research procedure using internationally accepted methods, different ultrasound doctors had slight operational differences that may have led to non-systematic errors[30]. This study only collected only data on pregnant women and foetuses from a hospital in Chongqing. The amount of data collected per gestational week was uneven. 


\section{Conclusions}

267 Overall, the foetal weight prediction equation established in this study can more accurately

268 predict foetal weight in Chongqing. Accurate prediction of foetal gestational age and weight can

269 be used to not only assess the growth and development of the foetus, but also guide the timing

270 and mode of delivery of a pregnancy. It can reduce the incidence of postpartum complications

271 and perinatal mortality, and provide a strong guarantee for the health of both mothers and

272 infants. Therefore, this equation is clinically valuable for monitoring and managing foetal weight.

273

274

275

276

277

278

279

280

281

282

283

284

285

286

\section{Declarations}

\section{Acknowledgements}

Not applicable.

\section{Funding}

Not applicable

\section{Availability of data and materials}

The data that support the findings of this study are available from the corresponding author LXD upon reasonable request.

\section{Ethics approval and consent to participate}




\section{Consent for publication}

Not applicable.

\section{Author details}

${ }^{1}$ Department of Obstetrics and Gynecology, the Second Affiliated Hospital of Chongqing Medical University,

Chongqing, Chongqing 400010, People's Republic of China.

${ }^{2}$ Chongqing Key Laboratory of Soft Condensed Matter Physics and Smart Materials, College of Physics, Chongqing

University, Chongqing, Chongqing 400030, People's Republic of China.

\section{Publisher's Note}

301 Springer Nature remains neutral with regard to jurisdictional claims in published maps and institutional affiliations. 
1. Verger C, Moraitis AA, Barnfield L et al (2020) Performance of different fetal growth charts in prediction of large-for-gestational age and associated neonatal morbidity in multiethnic obese population. Ultrasound in Obstetrics and Gynecology 56:73-77. http://doi.org/10.1002/uog.20413

2. Shah S NN, Hassan N (2015) Fetal Weight Estimation: Importance, Challenges and Emerging Trends. Annals Abbasi Shaheed Hospital \& Karachi Medical \& Dental College 20:148-154.

3. Shmueli A, Salman L, Hadar E et al (2019) Sonographic prediction of macrosomia in pregnancies complicated by maternal diabetes: finding the best formula. Archives of Gynecology \& Obstetrics. 299:97-103.

4. Milner J, Arezina J (2018) The accuracy of ultrasound estimation of fetal weight in comparison to birth weight: A systematic review. Ultrasound 26:32-41. http://doi.org/10.1177/1742271X17732807

5. Kiserud T, Piaggio G, Carroli G et al (2017) The World Health Organization Fetal Growth Charts: A Multinational Longitudinal Study of Ultrasound Biometric Measurements and Estimated Fetal Weight. PLOS 151:333-337. http://doi.org/10.1016/0002-9378(85)90298-4 volume. Obstetrics \& Gynecology 82:365-370. http://doi.org/10.1016/0378-5122(93)90009-7 
331 9. Stirnemann J, Villar J, Salomon L J et al (2017) International estimated fetal weight standards of the INTERGROWTH-21st Project. Ultrasound in Obstetrics \& Gynecology 49:478-486. http://doi.org/10.1002/uog.17347

10. Barel O, Vaknin Z, Tovbin J, Herman A, Maymon R (2013) Assessment of the Accuracy of Multiple Sonographic Fetal Weight Estimation Formulas: a 10-year experience from a single center. Journal of

11. Buck Louis G M, Grewal J, Albert P S, et al (2015) Racial/ethnic standards for fetal growth: the NICHD Fetal http://doi.org/10.1016/j.ajog.2015.08.032

12. Clark D C (2018) Association of Dairy Protein Intake During Pregnancy with Birth Weight. Food and Nutrition

13. Enhesari A, Fahimi F, Tahmouresi $\mathrm{H}$ (2018) The association between ultrasonic estimated fetal weight and neonatal weight. Journal of Research in Medical and Dental Sciences 6:35-38.

14. Shen Y, Zhao W X, Lin J H, Liu F S (2017) Accuracy of sonographic fetal weight estimation prior to delivery in a Chinese han population. Journal of Clinical Ultrasound 45: 465-471. http://doi.org/10.1002/jcu.22463

15. Woo J S, Li D F, Ma H K (1986) Intrauterine growth standards for Hong Kong Chinese. Australian and New

16. Luyao J, Shi L, Weijia X (2020) The impact of population and Business Vitality on Regional Economic Vitality-Take Chongqing as an Example. International Core Journal of Engineering 6:33-41.

17. Xue Z, Yinyin X, Hua Z, et al (2019) Birth weight charts for a Chinese population: an observational study of 
354 18. Meng L, Xu H, Liu A, et al (2013) The Cost and Cost-Effectiveness of a School-Based Comprehensive Intervention Study on Childhood Obesity in China. PLOS ONE 8:e77971. http://doi.org/10.1371/journal.pone.0077971

19. Hediger M L, Fuchs K M, Grantz K L, et al (2016) Ultrasound Quality Assurance for Singletons in the National 35:1725-1733. http://doi.org/10.7863/ultra.15.09087

20. Perni S C, Chervenak F A, Kalish R B, et al (2004) Intraobserver and interobserver reproducibility of fetal biometry. Ultrasound in Obstetrics \& Gynecology 24:654-658. http://doi.org/10.1002/uog.1717

21. Salomon L J, Alfirevic Z, Costa F D S, et al (2019) ISUOG Practice Guidelines: ultrasound assessment of fetal biometry and growth. Ultrasound in Obstetrics \& Gynecology 53:715-723. http://doi.org/10.1002/uog.20272

22. Salmon L J, Alfirevic Z, Berghella V, et al (2011) Practice guidelines for performance of the routine mid-trimester fetal ultrasound scan. Ultrasound in Obstetrics \& Gynecology 37:116-126.

23. Skupski D W, Owen J, Kim S, et al (2017) Estimating Gestational Age From Ultrasound Fetal Biometrics. Obstetrics and Gynecology 130:433-441.

24. Robinson R, Walker K F, White V A, Bugg G J, Snell K I, Jones N W (2020) The test accuracy of antenatal fetuses. Acta Obstet Gynecol Scand 99:503-509. 
27. Verfaille V, de Jonge A, Mokkink L, et al (2017) Multidisciplinary consensus on screening for, diagnosis and management of fetal growth restriction in the Netherlands. BMC Pregnancy and Childbirth 17:353. http://doi.org/10.1186/s12884-017-1513-3

28. Gardosi J, Francis A, Turner S, Williams M (2018) Customized growth charts: rationale, validation and clinical benefits.

American

$$
\text { Journal of }
$$

Obstetrics

and Gynecology

218:S609-S618.

29. Vetterlein J, Doehmen C, Voss H, et al (2021) Antenatal risk prediction of shoulder dystocia: influence of diabetes and obesity: a multicenter study. Archives of Gynecology \& Obstetrics 304:1169-1177. http://doi.org/10.1007/s00404-021-06041-7

30. Krispin E, Dreyfuss E, Fischer O, et al (2020) Significant deviations in sonographic fetal weight estimation: 


\begin{tabular}{ccccc}
\hline Variable & Estimate & SE & $P$-value & $R^{2}$ \\
\hline Intercept & 3.002741 & 0.041 & \\
BPD & -0.008753 & 0.001 & \\
HC & -0.000884 & 0.000 & \\
AC & 0.003206 & 0.000 & \\
FDL & -0.002894 & 0.001 & \\
BPD & 0.000005944 & 0.001 & \\
HC $^{2}$ & 0.00000222 & 0.001 & \\
AC $^{2}$ & -0.000002078 & 0.000 & \\
FDL $^{2}$ & 0.00004262 & 0.000 & \\
\hline
\end{tabular}

398 SE, standard error; BPD, biparietal diameter; HC, head circumference; AC, abdominal circumference; FDL, femur

399 diaphysis length; a $P$-value of less than 0.05 indicates statistical significance. $R^{2}$ is based on one randomly selected

400 sample for each participant.

401

402

403

404

405

406

407

408 
Table 2 The four equations

\begin{tabular}{lclll}
\hline Model & Sample & Scope & Race & Creation method \\
\hline Chongqing & 1925 & Regional & Single & Compensation mechanism \\
Hadlock & 167 & Regional & Single & Stepwise regression \\
Combs & 865 & Regional & Multiple & Multiple linear regression \\
Stirnemann & 2404 & Multicentre & Multiple & Second-degree fractional polynomial \\
\hline
\end{tabular}

410

411

412

413

414

415

416

417

418

419

420

421

422

423

424

425 


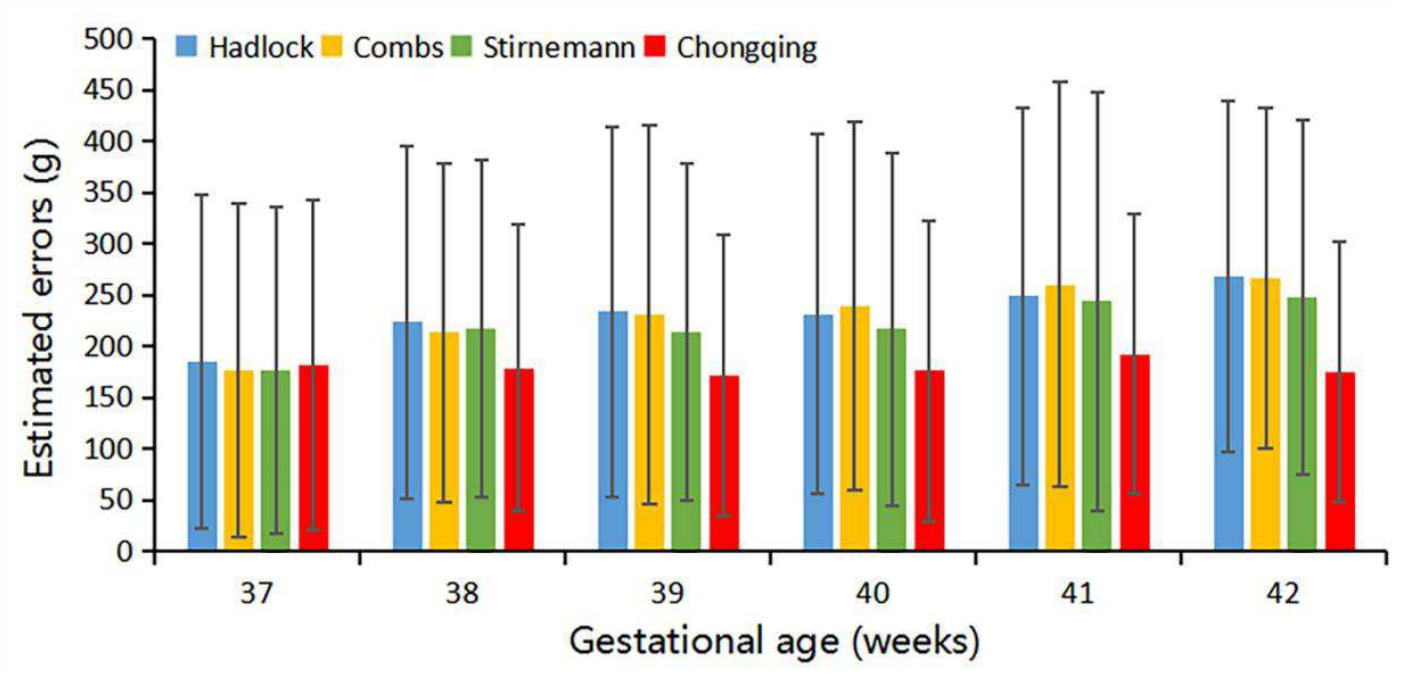

426

427

428

429

430

431

432

433

434

435

436

437

438

439

Figure 1 Comparison of estimated errors between estimated foetal weight and compensated weight for each gestational week 


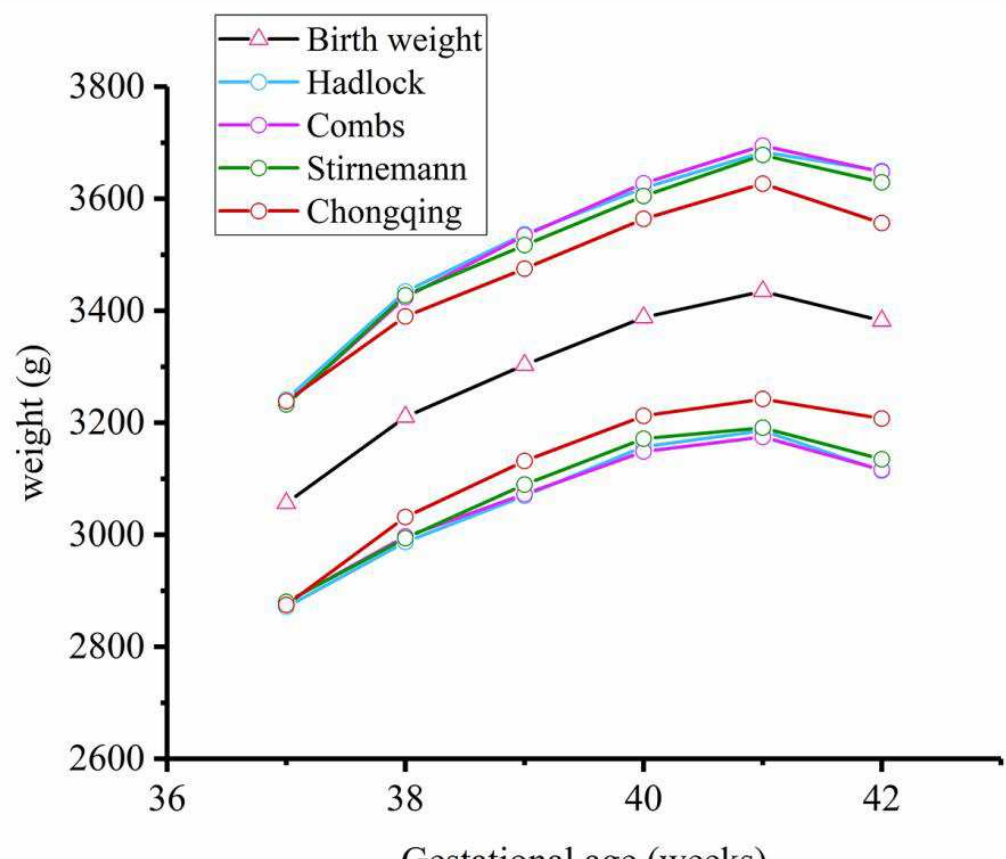

Gestational age (weeks)

442 Figure 2 Comparison of estimated errors between estimated foetal weight and birth weight for 
Table 3 Comparison of estimation error in the establishment set

\begin{tabular}{cccccc}
\hline Estimation error & In this study & Hadlock & Combs & Stirnemann & $P$ \\
Mean absolute error & 178.9 & 234.1 & 236.3 & 221.3 & $<0.001$ \\
Standard deviation & 140.3 & 178.0 & 182.9 & 176.1 & $<0.001$ \\
\hline
\end{tabular}

456

A $P$-value of less than 0.05 indicates statistical significance.

457

458

459

460

461

462

463

464

465

466

467

468

469

470

471

472

473 
Table 4 Comparison of estimation error in the validation set

\begin{tabular}{cccccc}
\hline Estimation error & In this study & Hadlock & Combs & Stirnemann & $P$ \\
Mean absolute error & 173.1 & 180.7 & 207.2 & 188.2 & $<0.001$ \\
Standard deviation & 128.6 & 143.8 & 152.8 & 148.6 & $<0.001$ \\
\hline
\end{tabular}

475

A $P$-value of less than 0.05 indicates statistical significance.

476 